\title{
Coordinated Optimization of Emergency Power Vehicles and Distribution Network Reconfiguration Considering the Uncertain Restoration Capability of E-taxis
}

\author{
Lu Zhang, Member, IEEE, Shujun Jiang, Bo Zhang, Gen Li, Member, IEEE, Zhaoqi Wang, Wei \\ Tang*, Member, IEEE
}

\begin{abstract}
Network reconfiguration and emergency power vehicles (EPVs) dispatching are widely used in distribution networks for load restoration. However, their capabilities are limited by the allocated amounts of circuit breakers and EPVs. Etaxis can also participate in the restoration as a kind of mobile energy storage using the vehicle to grid (V2G) technology. However, the uncertainty of E-taxis should be considered in the restoration. To achieve better effectiveness of the restoration and fully utilize the capability of network reconfiguration, EPVs and E-taxis, this paper proposes a coordinated restoration optimization method considering the uncertain restoration capabilities of discharging stations with E-taxis. A joint probability distribution function is established based on Gaussian Mixture Model to describe the uncertainty of station discharging capabilities considering the correlation of user rationality, taxi state-of-charge and transportation status. Then, a bi-level programming model embedded with the chance constraint programming is developed to optimize the coordinated dynamic restoration scheme of the network reconfiguration and EPV dispatching, with the consideration of the mobility of EPVs during the restoration. Simulations studies are performed to verify the proposed method.
\end{abstract}

Index Terms - Restoration, distribution network, emergency power vehicle, network reconfiguration, E-taxis, Gaussian Mixture Model.

\section{INTRODUCTION}

The fault recovery capabilities of urban distribution networks need to be increased to meet the high requirements for the continuous load power supply [1], [2].

Network reconfiguration has been widely used in distribution networks for load transfer between feeders to achieve load restoration [3]. However, the effectiveness of the restoration is affected by the allocation of circuit breakers and switching strategy, as well as the power transfer capability of the adjacent feeders. Therefore, although network reconfiguration has a

This work was supported by Funds for International Cooperation and Exchange of the National Natural Science Foundation of China (Grant No. 52061635104) . (Corresponding author: Wei Tang)

Lu Zhang, Bo Zhang, Zhaoqi Wang and Wei Tang are with China agricultural university, Beijing, 100083, China (e-mail: zhanglu1@ @au.edu.cn; zhangbo1223@foxmail.com; seven_wzq@cau.edu.cn; wei_tang@cau.edu.cn). strong capability, it may be inflexible due to the limitations of the switches, e.g. their locations, number and switching constraints. Emergency power vehicles (EPVs) [4], [5] are also widely used in some developed cities for point-to-point power supply during a power restoration. However, due to the high capital cost of EPVs, they are not widely used in the post-fault restoration of distribution networks.

Vehicle-to-grid (V2G) technology has been proposed to achieve the power supply of electric vehicles (EVs), which can also support the power grid restoration by using EVs as mobile energy storage devices [6]-[8]. Electric taxis (E-taxis) exhibit flexibility and feasibility to participate in the power restoration. This is because that there are large amounts of E-taxis in the transportation system and they can obey the service agreement with the taxi company. A large number of E-taxis discharging to the grid may be able to provide enough electricity for the restoration of the distribution network. Moreover, the mobility of E-taxis in the traffic network can ensure a quick response speed and flexible restoration scheme for loads with different critical levels. However, the uncertainty of E-taxis cannot be neglected during such a restoration dispatch.

Various restoration methods have been proposed in the literature. Reference [9] presents a multi-stage restoration method combining the island operation of DGs and network reconfiguration to maximize the restoration of loads. To cope with the uncertainty of DGs, reference [10] proposes a robust islanded restoration strategy. However, only emergency EVs have been modeled in this work. Conventional uncertainty optimization method, robust optimization [11]-[12] and distributional robust optimization [13]-[14] are used to deal with the uncertainty of EV and DG. An improved water evaporation optimization algorithm has been proposed in [15] to solve the multi-constraint optimization problem of coordination of DGs, battery energy storage systems and network reconfiguration. Reference [16] proposes an

Shujun Jiang is with the University of Manchester, Manchester, M13 9PL, U.K. (e-mail: shujunjsj@gmail.com)

Gen Li is with Cardiff University, Cardiff, CF24 3AA, U.K. (e-mail: LiG9@cardiff.ac.uk). 
optimization strategy for network reconfiguration considering the centralized EV charging stations as the black-start power sources. However, the EVs in the traffic network are neglected.

There are two main shortcomings of the existing work considering the uncertainties of EVs in the restoration of distribution networks.

1) The distribution of E-taxis in traffic networks and the response behavior of users exhibit high uncertainties. Since the charging station is the interface of EVs and distribution networks, it inherits the uncertainty of EVs and pass this uncertainty in the power supply in real time. Meanwhile, the correlation of power supply capabilities between multiple charging stations must be considered to guarantee the description accuracy of the uncertainty. However, most of the existing research fails to consider the correlation of these uncertain variables, or they are too complex to be embedded in optimal restoration models. Therefore, the uncertainty of the power supply capability of EV stations must be carefully described and embedded in the optimization model.

2) Effectiveness of the load restoration can be further improved by the coordination between network reconfiguration and EPV dispatching strategies considering the uncertain restoration capability of E-taxis. The flexibility of EPVs can enhance the power supply capability of distribution networks, which will influence the optimal results of network reconfiguration, although the discharging capability of EPVs is uncertain. Therefore, how to coordinately optimize the network reconfiguration and island segmentation with EPV dispatching under an uncertain restoration capability of E-taxis must be considered.

The paper proposes a coordinated restoration method using the network reconfiguration and EPVs with the consideration of the uncertain restoration capabilities of discharging stations with E-taxis. A joint probability distribution function is established based on Gaussian Mixture Model (GMM) to describe the uncertainty of station discharging capabilities considering the correlation of user rationality, taxis' state-ofcharge (SoC) and transportation influences. A bi-level programming model embedded with the chance constraint programming is developed to optimize the coordinated dynamic restoration scheme of network reconfiguration and EPV dispatching considering the mobility of EPV during the restoration period.

The contributions of this paper are as follows:

1) To better characterize the uncertainty of capability of charging/discharging stations, a Gaussian Mixture Model is proposed to describe the uncertainty of the E-taxis including the factors of the user rationality, taxi state-of-charge and transportation status.

2) E-taxi and conventional methods (EPVs and reconfiguration) are coordinated to achieve a better effect of load restoration. The chance constraint programming is embedded in the bi-level programming model to cope with the uncertainty induced by E-taxis.

\section{II.PROBLEM DESCRIPTION}

Fig. 1 shows a 5-feeder urban distribution network, wherein some power lines have been damaged and the utility grid can only restore part of loads through network reconfiguration. Etaxis support the network through the charging pile in charging station, and EPVs restore the network node point to point. EPVs belong to and are fully controlled by the power supply company, whose obligation is for load restoration. E-taxis belong to the taxi company that cannot be dispatched by the power supply company directly. Three EPVs are standby at Node 16 and available to be dispatched for load restoration, which can achieve an 8-hour power supply with $200 \mathrm{~kW}$. Several charging/discharging stations are allocated at Nodes 43 and 64 with 25 discharging piles separately, whose rated power is 40 $\mathrm{kW} .200 \mathrm{E}$-taxis with $80 \mathrm{kWh}$ rated power battery are cruising around, whose discharging power is the same as the connected charging pile. It is assumed that taxi drivers are able to receive the dispatching suggestions for load restoration, and some of them will decide whether to go to the nearby charging/discharging stations, whose restoration capabilities are uncertain considering the driver behavior, taxi SoC and traffic conditions. A coordinated optimization method is therefore needed to optimize the restoration scheme of using network reconfiguration and EPVs considering the uncertain restoration capability of taxis during the restoration period. However, EPV dispatching is dependent on the restoration topology which is determined by network reconfiguration, and also affected by the uncertain restoration capability of discharging stations because the waste of energy may happen if EPVs and taxis are serving in the same area. Moreover, to achieve an optimal distribution network restoration, the optimization of the network reconfiguration must consider both EPV dispatching and the uncertain restoration capability of discharging stations which are powered by E-taxis. Therefore, a bi-level programming model embedded with chance constraint programming is developed, which is utilized to realize the coordinated optimization of EPVs and network reconfiguration considering the uncertainty of station discharging capabilities. 


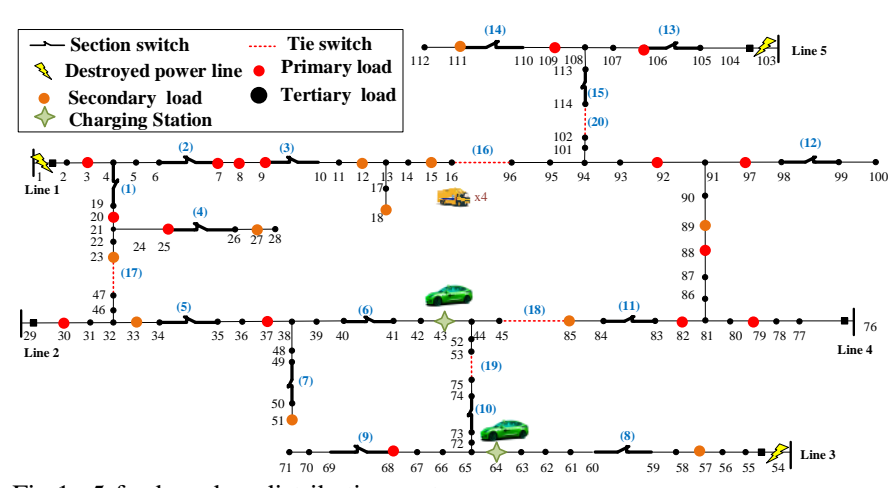

Fig 1. 5-feeder urban distribution system. layers.

\section{UNCERTAIN CAPABILITY MODELING OF DISCHARGING STATIONS BASED ON GMM}

\section{A. Modeling of E-taxi Restoration Capability}

Historical transportation data of E-taxis is needed to describe the probability distribution functions. That is because the realtime locations of E-taxis are unknown due to privacy [17]. Thus, three parameters of E-taxis are used to model their response capabilities of restoration, which are E-taxis' positions in the traffic network, current moving directions and SoCs of their batteries. For only one E-taxi, its parameter set in the navigation map of the traffic network can be expressed as:

$$
\Gamma=\{\text { Dir }, L, \text { Dist }, \text { SoC }, t\}
$$

where Dir is the direction of motion of the E-taxi (Dir $=1$ means the direction of the E-taxi is consistent with the direction of the directed graph, Dir $=-1$ means the direction of the E-taxi is opposite to the direction of the directed graph), $L$ is the directed edge number of the road where the E-taxi is located, Dist is the distance between the E-taxi and the head vertices of the edge, $t$ is the time index, $\mathrm{SoC}$ is the state-of-charge of E-taxi at time $t$. The input data of the multi-dimensional GMM includes 24-hour E-taxis distributions in the transportation network, which contains time information (including peak hours). By calculating the conditional probability of the time dimension of GMM, the probability distribution functions (PDFs) can be obtained for each period.

Considering the current traffic conditions, the travel time of the E-taxi in street $k$ is as follows [18], [19]:

$$
T_{k, t}=\frac{l_{k}}{v_{k}^{\text {free }}}\left(1+R D I_{k, t}\right),
$$

where $l_{k}$ is the length of street $k ; v_{k}^{\text {free }}$ represents the free-flow speed in street $k ; R D L_{k, t}$ is the relative delay index of street $k$ at time $t$ which quantifies the percentage increase when it is moving due to the traffic congestion on the street, which can be obtained from the real-time traffic database.

Dijkstra algorithm [20] is used to select the path with the minimum time consumption for the E-taxis.

$$
\begin{gathered}
t_{i k}=t_{0}+\sum_{c \in \text { path }_{i k}} T_{k, t}, \\
\operatorname{SoC}\left(t_{i k}\right)=\operatorname{SoC}\left(t_{0}\right)-\sum_{c \in \text { path }_{i k}} l_{c} \times e_{s}, \\
\operatorname{SoC}\left(t_{i k}\right)>\operatorname{SoC}_{\text {min }}+P_{c}^{E T} \Delta t,
\end{gathered}
$$

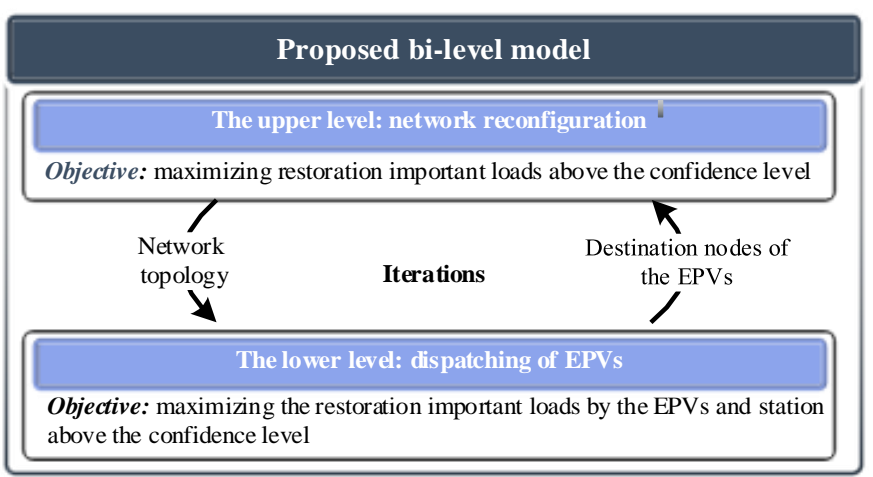

Fig 2. The logical relationship between the upper and lower

$$
\sum_{c \in \text { path }_{i k}} T_{k, t}<\Delta T-\Delta t
$$

where $t_{i k}$ is the arriving time of the $i_{t h}$ E-taxi at the $k_{t h}$ charging station, $t_{0}$ is the time of accepting the response order, path $_{i k}$ is the path from the E-taxi to the station using the shortest time, $l_{c}$ is the length of street $c, e_{s}$ is the power consumption per kilometer, $S o C_{\min }$ is the minimum SoC considering the battery health, $P_{c}^{E T}$ is the discharge power of E-taxi, $T$ is the restoration period, $\Delta t$ is the time step of dispatching period, where $T=n \Delta t$.

E-taxi drivers are not perfectly rational. The reward of Etaxis who participate in $\mathrm{V} 2 \mathrm{G}$ response depends on the discharging capacity as the agreement set a margin price for unit discharging [21]. It is assumed that the probability that drivers agree or disagree with participating in the $\mathrm{V} 2 \mathrm{G}$ response is normally distributed.

After receiving the instruction, drivers that agree to participate in the restoration will drive to the nearest charging stations. The parameter set of the E-taxi for charging station $i$ will be updated to:

$$
\Gamma_{i, E T}=\left\{S o C_{r m}, t_{r m}\right\}
$$

where $S o C_{r m}$ is the SoC of the E-taxi after arrival obtained from (5), $t_{r m}$ is the arrival time obtained from (3).

\section{B. Modeling of the Capability of Discharging Stations during the Restoration}

The 5-feeder urban distribution system is modified based on a real distribution network, in which the charging stations have been determined and assumed that the stations have the V2G technology and are available to achieve discharging. The Etaxis (including in-transit and in-station E-taxis) to a certain charging station $i$ can be used as energy storage activated at a specified time, represented as the set $\Gamma_{i, E T}$. The E-taxis which meet the following discharge requirement in the station are allowed to participate in the response:

$$
S o C_{r m} \geq S o C_{\text {min }}+P_{c}^{E T} \Delta t
$$

The SoC condition of an E-taxi during the discharging process is shown in Fig. 3. $P_{c}^{E T} \Delta t$ is the charging demand in a unit period, $t_{d i s}^{s t}$ is the start time of discharging, and $t_{d i s}^{\text {end }}$ is the end time of discharging. As E-taxis belong to the same company, the discharging time of E-taxi could be calculated simply by dividing the SoC (multiply by $98 \%$ ) by the discharging power. 


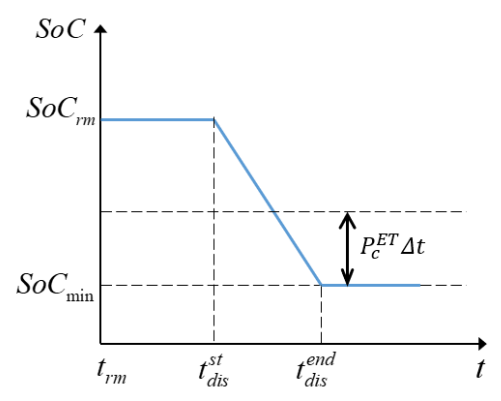

Fig. 3. Discharging SoC condition.

If $\Sigma P_{i, t}^{E T}>\Sigma P_{\max }$, which means all the charging piles of the station are being used at maximum power, the rest of the E-taxis will wait according to their arrival time. Their parameters are updated as: $t_{r m+\Delta t}=t_{r m}+\Delta t, S o C_{r m+\Delta t}=S o C_{r m}$.

The charging stations within the isolated islands become the power source after the fault. As one E-taxi can only go to one charging station, therefore the more E-taxis go to Station A the fewer E-taxis will go to Station B. It means that there is a correlation between different charging stations, and the discharge power of them at the same time are not independent random variables. Then, the Gaussian mixture model is used to fit the probability distribution of the discharge power in charging stations, and its covariance matrix represents the correlation between data of different dimensions.

If there are $W$ charging stations on the island, the discharge power $\Gamma_{\Sigma W P}$ of them can be expressed as:

$$
\begin{gathered}
\Gamma_{\Sigma i, P}=\left[\sum P_{i, t_{0}}^{E T}, \sum P_{i, t_{0}+\Delta t}^{E T}, \sum P_{i, t_{0}+2 \Delta t}^{E T} \ldots \sum P_{i, t_{0}+T}^{E T}\right]^{T}, i \in M, \\
\Gamma_{\Sigma W P}=\left[\Gamma_{\Sigma 1, P}, \Gamma_{\Sigma 2, P}, \ldots, \Gamma_{\Sigma w, P}\right] .
\end{gathered}
$$

The corresponding time dimension is:

$$
t_{E T}=\left[t_{0}, t_{0}+\Delta t, t_{0}+2 \Delta t, \ldots, t_{0}+T\right]^{T} \text {. }
$$

The joint GMM is built based on random variable $P$ :

$$
P=\left[\begin{array}{ll}
t_{E T} & \Gamma_{\Sigma W P}
\end{array}\right] \text {. }
$$

The GMM PDF for $W+1$ dimension random variables $P$ are:

$$
\begin{array}{r}
f_{P}(P)=\sum_{m=1}^{M} \omega_{m}^{P} N_{m}^{P}\left(P ; \mu_{m}^{P}, \Sigma_{m}^{P}\right), \\
F_{P}(P)=\sum_{m=1}^{M} \omega_{m}^{P} F\left(P ; \mu_{m}^{P}, \Sigma_{m}^{P}\right),
\end{array}
$$

where $f_{P}(P)$ and $F_{P}(P)$ are the joint PDF and CDF of multivariate $P, M$ is the number of GMM components; $\omega_{m}^{P}$ is the weighed coefficient; $N_{m}^{P}\left(P ; \mu_{m}^{P}, \Sigma_{m}^{P}\right)$ is the multivariate Gaussian distribution.

To analyze the optimization model, the conditional probability invariance of GMM [22] is used to obtain the probability distribution of the injected power of a certain charging station at a given time:

$$
\begin{aligned}
& f_{\left.P_{t_{E T}, m} \mid t_{E T}, m\right)}\left(P_{t_{E T}, m} \mid\left(t_{E T}, m\right)\right)= \\
& \sum_{m=1}^{M} \omega_{m}^{\prime} N_{m}^{\prime}\left(P_{t_{E T}, m} \mid\left(t_{E T}, m\right) ; \mu_{m}^{P_{t E T, m} \cdot\left(t_{E T}, m\right)}, \Sigma_{m}^{P_{t E T}, m} P_{t E T, m} \cdot\left(t_{E T}, m\right)\right)^{\prime} \\
& F_{\left.P_{t_{E T}, m} \mid t_{E T}, m\right)}\left(P_{t_{E T}, m} \mid\left(t_{E T}, m\right)\right)=
\end{aligned}
$$

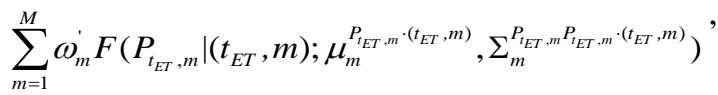

where $f_{P_{I_{E T}, m} \mid\left(t_{E T}, m\right)}$ and $F_{P_{t E T}, m} \mid\left(t_{E T}, m\right)$ are the PDF and CDF of the injected power of Station $m$ at time $t_{E T}$.

\section{Multi-state Model}

The discharge power of the charging station follows the GMM distribution $f_{P_{t, m} \mid(t, m)}$, the abscissa value of which is from 0 to $\Sigma P_{\max }$. Dividing the internal $\left[0, \Sigma P_{\max }\right.$ ] into $N_{c}$ states. $P_{c}(i)$ represents the discharge power of a certain station in state $i$, and $F_{c}(i)$ is its probability. They can be calculated as follows:

$$
\begin{gathered}
P_{c}(i)=\left[\left(i-\frac{1}{2}\right) / N_{c}\right] \cdot \sum P_{\max }, \\
F_{c}(i)=\int_{\left[(i-1) / N_{c}\right] \sum P_{\max }}^{\left(i / N_{c}\right) \cdot \sum P_{\max }} f_{P_{t, m} \mid(t, m)} d P_{t, m},
\end{gathered}
$$

where $i=1,2, \cdots, N_{c}$.

\section{Optimal Restoration Method BASEd ON Bi-LeVel MODEL EMBEDDED WITH CHANCE CONSTRAINT PROGRAMMING}

The diagram of the proposed bi-level model embedded with chance constraint programming is shown in Fig. 4. In the upper level, the network topology is optimized with the objectives of maximizing the restoration amount of important loads above the confidence level, according to the dispatching results of EPVs and probability distribution of discharge power of the charging station. In the lower level, the destination nodes of the EPVs are optimized considering the probability distribution of discharge power of the charging station, to maximize the restoration amount of important loads by the EPVs and charge station above the confidence level. Firstly, the upper level initializes network topology subjected to the constraints of the upper level and transfers them to the lower level. Secondly, the lower level optimizes the destination nodes of the EPVs based on the network topology of the upper level and returns the dispatching results and corresponding objective to the upper level. Then, the upper level evaluates the restoration results, and regenerates a new network topology, which will be returned to the lower level. Finally, stable restoration schemes will be obtained by iterations between the two levels.

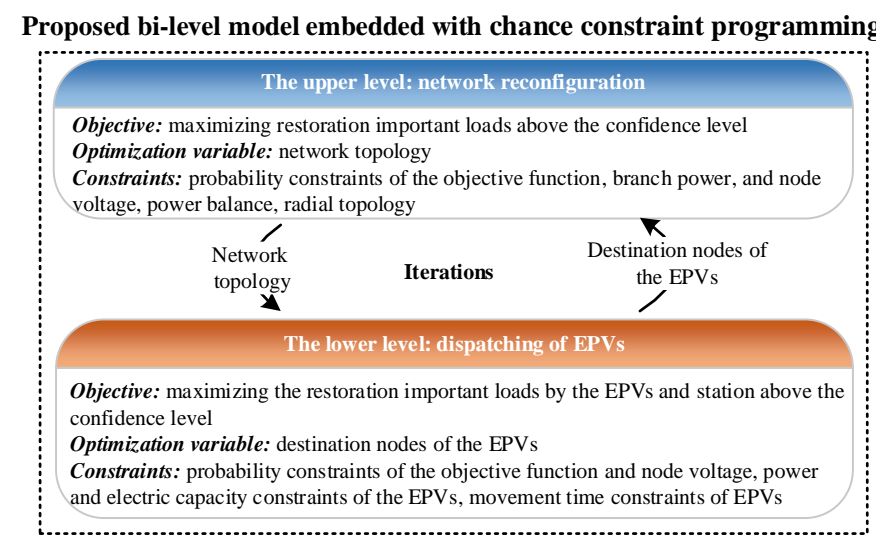

Fig. 4. The diagram of the bi-level model.

\section{A. Optimization Model of Upper Level}

Restoring as much important load as possible is the most important objective during the restoration period. To consider 
the uncertain restoration capability of the charging station, the upper level model takes the maximum restoration amount of important loads above the confidence level as the objective function as shown in (19).

$$
\max \overline{F_{U}}
$$

Moreover, the optimization model of the upper level is subjected to the following constraints.

1) Probability constraints of the objective function

$$
\begin{gathered}
\sum_{i=1}^{\xi_{\max }} P\left\{f\left(X_{1}, \xi_{i}\right) \geq \overline{F_{U}}\right\} \geq \alpha, \\
f\left(X_{1}, \xi\right)=F_{\text {main }}+F_{\mathrm{ET}}+F_{E P V}, \\
F_{\text {main }}=\sum_{t=1}^{T} \sum_{i \in R} \lambda_{i} L_{i, t} y_{i, t}, \\
F_{\mathrm{ET}}=\sum_{t=1}^{T} \sum_{i \in D} \lambda_{i} L_{i, t} y_{i, t}, \\
F_{E P V}=\sum_{t=1}^{T} \sum_{i \in D} \lambda_{i} L_{i, t} y_{i, t},
\end{gathered}
$$

where $X_{1}$ is the decision variable of the upper level, i.e. switch state, $\xi_{i}$ is the $i$ th state, $\xi_{\max }$ is the maximum number of states, $f\left(X_{1}, \xi\right)$ is the maximum restoration amount of important loads, $\alpha$ is the confidence level, $\overline{F_{U}}$ is the maximum value of $f\left(X_{1}, \xi\right)$ when the confidence level is not lower than $\alpha, P\{\bullet\}$ is the probability, $F_{\text {main }}$ is the amount of load restored by the main grid during the restoration period, $F_{\mathrm{ET}}$ and $F_{\mathrm{EPV}}$ are the restoration amount of load by the E-taxis and EPVs during the restoration period, $T$ is the fault duration, $R$ is a set of outage area, $D$ is a set of outage area after reconfiguration, $\lambda_{i}$ is the importance of the load at node $i, L_{i, t}$ is the load at node $i$ at time $t$ and $y_{i, t}$ is state parameter $\left(y_{i, t}=1\right.$ means node $i$ at time $t$ has been restored the power supply, $y_{i, t}=0$ means node $i$ at time $t$ has not been restored).

2) Probability constraints of the branch power

$$
P\left\{P_{l}\left(x_{1}, \xi\right) \leq P_{l}^{\max }\right\} \geq \beta_{p},
$$

where $P_{l}\left(x_{1}, \xi\right)$ and $P_{l}^{\text {max }}$ are the active power in branch $l$ under state $\xi$ and allowable maximum active power and $\beta_{p}$ is the confidence level of the branch power.

3) Probability constraints of the node voltage

$$
P\left\{U_{i}^{\min } \leq U_{i}\left(x_{1}, \xi\right) \leq U_{i}^{\max }\right\} \geq \beta_{U},
$$

where $U_{i}\left(x_{1}, \xi\right)$ is the node $i$ voltage under state $\xi, U_{l}^{\max }$ and $U_{l}^{\min }$ are the allowable maximum and minimum voltages and $\beta_{U}$ is the confidence level of the node voltage.

4) Power balance constraints

$$
\begin{aligned}
& P_{i, t}-V_{i, t} \sum_{j=1}^{n} V_{j, t}\left(G_{i j} \cos \theta_{i j, t}+B_{i j} \sin \theta_{i j, t}\right)=0, \\
& Q_{i, t}-V_{i, t} \sum_{j=1}^{n} V_{j, t}\left(G_{i j} \sin \theta_{i j, t}-B_{i j} \cos \theta_{i j, t}\right)=0,
\end{aligned}
$$

where $P_{i, t}$ and $Q_{i, t}$ are injection active and reactive power of node $i$ at time $t, P_{i j}$ and $B_{i j}$ are the real and imaginary parts of node admittance matrix, $\theta_{i j}$ is the phase angle difference between node $i$ and $j$ at time $t, n$ is the total number of nodes.

5) Radial topology constraint

$$
N_{r e} \in N
$$

where $N_{r e}$ is the network topology after reconfiguration, $N$ is the set of radial topologies. According to reference [23], it is possible to ensure that the distribution network corresponds to a spanning tree connected to the main substation, regardless of the direction of power flow, by introducing two binary variables corresponding to each line [as described in [24] by (11)-(15)].

\section{B. Optimization Model of Lower Level}

In the lower level, the destination nodes of the EPVs are optimized considering the probability distribution of discharge power of the charging station and will, in turn, affect the reconfiguration results of the main grid. The objective function of lower-level optimization is to maximize the restoration amount of important loads by the EPVs and charging stations above the confidence level.

$$
\max \overline{F_{L}}
$$

The constraints need to be satisfied as follows. 1) Probability constraints of the objective function

$$
\begin{gathered}
\sum_{i=1}^{\xi_{\max }} P\left\{f\left(X_{2}, \xi_{i}\right) \geq \overline{F_{L}}\right\} \geq \alpha, \\
f\left(X_{2}, \xi\right)=F_{\mathrm{ET}}+F_{E P V},
\end{gathered}
$$

where $X_{2}$ is the decision variable of the lower level, $\overline{F_{L}}$ is the maximum value of $f\left(X_{2}, \xi\right)$ when the confidence level is not lower than $\alpha$.

2) Power and electric capacity constraints of the EPVs

$$
\begin{aligned}
P_{i, t}^{E P V} & \geq L_{i, t}, \\
\operatorname{SoC}_{i, t}^{E P V} & \geq L_{i, t} y_{i, t} \Delta t,
\end{aligned}
$$

where $P_{i, t}^{E P V}$ is the discharge power of EPVs on node $i$ at time $t$, $S o C_{i, t}^{E P V}$ is the SOC of the EPVs on node $i$ at time $t, L_{i, t} y_{i, t} \Delta t$ is the required electric quantity of load on node $i$ during the restoration.

3) Probability constraints of the node voltage in the restored by charging station

$$
P\left\{V_{\min }<V_{i, t}^{E T s^{\prime}}<V_{\max }\right\} \geq \beta_{U},
$$

where $V_{i, t}^{E T S}$ is the voltage of node $i$ restored by charging station at time $t$.

\section{4) Movement time constraints of EPVs}

Due to the uncertainty of station discharging capabilities, the discharge power fluctuates during the restoration and the restoration area changes accordingly. Therefore, EPVs are allowed to move during the restoration period to make full use of the restoration capabilities of EPVs and taxis. The movement time of EPVs between any two nodes $i$ and $j$ is:

$$
T_{t, i j}=\sum_{k \in \text { pathij }} T_{k, t}=\sum_{k \in \text { pathij }} \frac{l_{k}}{v_{k}^{\text {free }}}\left(1+R D I_{k, t}\right) .
$$

where $R D L_{k, t}$ is the relative delay index of street $k$ at time $t$, which represents the degree of the traffic congestion. It should be noted that if the fault occurs in peak hours, the parameter $t$ of $R D L_{k, t}$ would be set accordingly. Moreover, the movement time of EPVs were set to an integer multiple of the scheduling period $\Delta t$, which is described in equation (37), based on which the traffic regulations and congestion were considered in the proposed method.

$$
T_{t, i j}=(x+1) \Delta t \text {, if } x \Delta t<T_{t, i j} \leq(x+1) \Delta t .
$$


In this paper, the EPVs were assumed belonging to a power supply company which is responsible for the load power supply under emergency restoration, and therefore, the cost of the EPVs will be covered by the power supply company.

\section{Algorithm of Bi-Level Model}

The upper level is to optimize the states of switches, which can be represent as a $(0,1)$-matrix. The binary codes are used in the particle swarm optimization (BPSO) [25] to represent the optimization variables. Therefore, the BPSO algorithm is selected for the upper level to optimize the switch states which are binary variables. The dispatching results of EPVs are optimized in the lower level which has lots of possible combinations. Moreover, the solution space is an exponential growth with the increase of distribution network nodes. The genetic algorithm (GA) with the elitist strategy [26] is used in the lower level because it can solve complex problems with lots of uncertain constraints [27]. Moreover, GA can avoid being trapped in local optimal solution and accelerate the convergence. After repeated iterations, the coordinated optimization of EPVs and network reconfiguration in the distribution network will achieve.

The calculation process of the proposed bi-level optimization model as follows.

Step 1: Set the initial parameters including the distribution system parameters, typical daily load, E-taxis and EPV parameters.

Step 2: Establish the joint probability distribution functions of the charging stations based on GMM and building the corresponding multi-state model in every period.

Step 3: Randomly generate the initial network topology that satisfies the upper level constraints. At the same time, the number of upper level iterations $K_{U}$ is set to 1 .

Step 4: First, for each network topology in the upper level, the lower level generates initial populations which is the destination nodes of EPVs and the number of lower level iterations $K_{L}$ is set to 1 . Second, carry out power flow calculation for each period and each state, and check the confidence level for constraint conditions. Then, an optimal individual in the lower level is obtained through the GA.

Step 5: Return the optimization results of the lower level including objective values and dispatching results of EPVs to the upper level.

Step 6: Calculate the upper level objective functions and check the confidence level for constraint conditions.

Step 7: Determine whether $K_{U}$ has reached the maximum allowable number of iterations $K_{U}^{\max }$ or not. If it reaches $K_{U}^{\max }$, the calculation will be finished, otherwise, the population of the upper level will be updated and go to Step 4 .

\section{V.CASE STUDIES}

Two studies have been conducted. The first one demonstrates the need of considering the uncertain restoration capability of discharging stations based on GMM. The second demonstrates the superiority of proposed coordinated restoration method.

\section{A. Simulation Set-up}

The 5 -feeder distribution system in Fig. 1 is used to demonstrate the proposed method, in which the maximum load power is $8543.99 \mathrm{~kW}+5918.77 \mathrm{kVar}$. The fault is set at $10 \mathrm{am}$ and lasts 4 hours and the dispatching time-scale in this paper is 0.5 hour. The confidence level is set as 0.8 . The charging and discharging efficiency of the battery of E-taxis is $98 \%$.

\section{B. Benefits for Considering the Uncertain Restoration Capability of Discharging Stations}

Case $\mathbf{1}$ is the scheme considering network reconfiguration and EPVs without E-taxis.

Case 2 is the scheme considering network reconfiguration, EPVs and E-taxis, but only the maximum discharging power is used in discharging stations without considering the uncertainties of taxis.

Case 3 is the scheme considering network reconfiguration, EPVs and E-taxis, but the E-taxis are assumed to arrive in the first 5 minutes. It means the uncertainty of the transportation status of E-taxis is neglected.

Case 4 is the scheme proposed in this paper, which considering network reconfiguration, EPVs and E-taxis, as well as the uncertainties of taxis.

Optimization results of restorations are shown in Table I and Fig. 5, while the restoration power capacities are shown in Table II. The recovery capacity of total and primary load power in Case 1 is $12629.78 \mathrm{kWh}$ and $4533.75 \mathrm{kWh}$, as shown in Table II, which is the lowest in the four cases. That is because the restoration capacities are limited by circuit breakers and EPV numbers. Thus primary loads cannot be fully recovered.

The recovery capacity of loads in Case 2 is increased to $312.48 \mathrm{kWh}$ supported by discharging stations, in which the restoration capability of Line 2 has been enhanced by the discharging Station 1 at Node 43, and therefore, loads 7, 8 and 9 are recovered. Loads $60-68$ and $72-73$ are restored by discharging Station 2, which achieve an island recovery as shown in Fig. 5(b). Therefore, $449.07 \mathrm{kWh}$ loads are further restored compared with Case 1. However, the actual restoration capability of discharging stations may be smaller than the ideal result in Case 2 because of an uncertain number of E-taxis. As shown in Fig. 6, the actual discharging power of the station at Node 43 is only $574 \mathrm{~kW}$ at 10:30 am. Thus, only loads 7 and 8 can be further restored by Line 2 , and the actual restoration island of discharging station 2 is Nodes 60-67 and 72, shown as the area with the solid line. Other loads are all shed including the primary load 68 . Therefore, the actual recovery capacity of primary loads during the whole restoration period is only $4669.82 \mathrm{kWh}$ in Case 2, as shown in Table II.

In Case 3, the recovery capacity for the total and primary load power is $15278.24 \mathrm{kWh}$ and $5174.725 \mathrm{kWh}$ if the transportation status uncertainty of E-taxis is neglected. However, the actual recovery capacity is $14733.33 \mathrm{kWh}$ and $4808.98 \mathrm{kWh}$ respectively. It is because the SoCs of E-taxis are higher than the actual situation due to the ignored power consumption on the road. Besides, insufficient uncertainty analysis overestimates the discharging ability of station 2, and EPV 
cannot move and support node 68 in time, which decreases the primary load recovery rate compared with Case 4.

The actual recovery capacity of the primary loads in Case 4 is $5005.34 \mathrm{kWh}$, as shown in Table II, which is $7.2 \%$ higher than that in Case 2. That is because the uncertain restoration capabilities of discharging stations have been considered in the coordinated optimization of network reconfiguration and EPV dispatching. The probability distribution functions of uncertain discharging capability for stations at 10:30 am are illustrated in Fig. 7, and the optimization methods of restoration are given in Table I. The discharging capabilities of stations in the last two periods are smaller due to the decreased SoCs of taxis, based on which load 9 is shed. Meanwhile, loads 68 and 73 which are supported by Station 2 are shed from period 7, while the EPV at Node 57 moves to the primary load 68 to improve restoration effects, as shown in Fig. 5(c). In Case 4, the primary load restored by EPVs is greater than that in Case 2, while the total load recovered is less than that in Case 2, which are shown in Table II. That is because the dispatching of EPVs compensates for the shed of the primary load caused by the uncertainty of the charging stations. However, it should be noted that the movement of EPVs needs time and therefore, results in a little decrease in the total load.

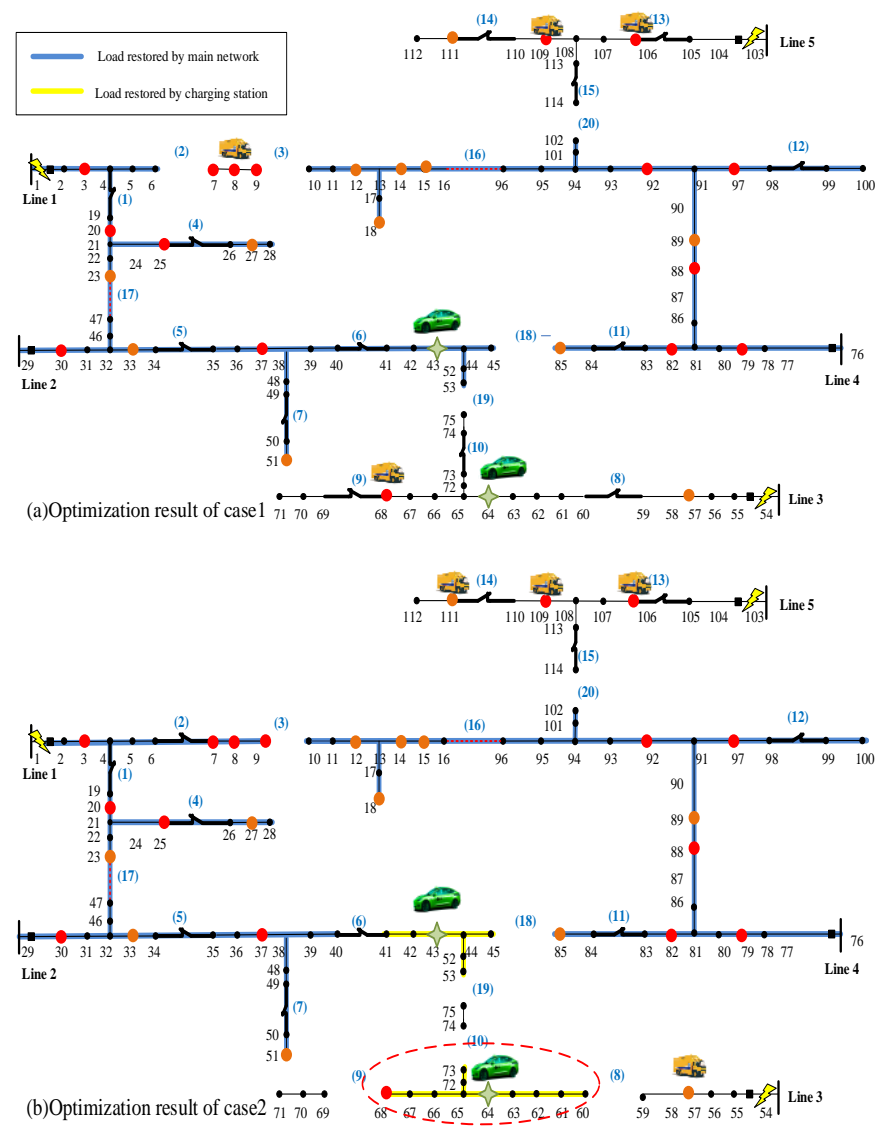

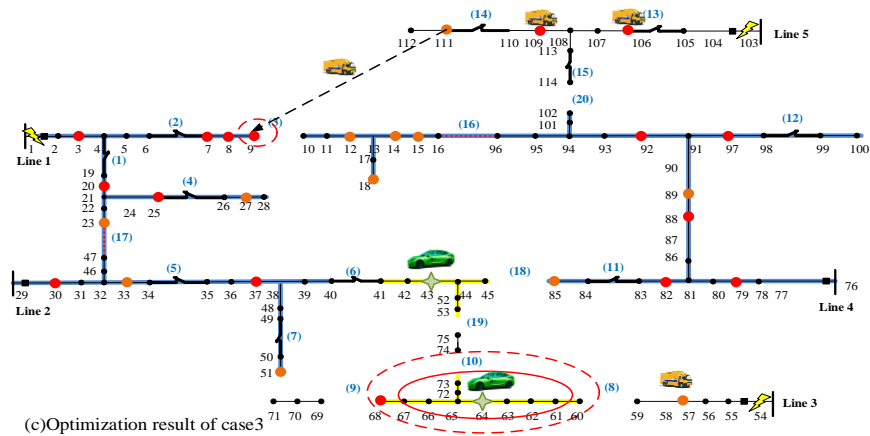

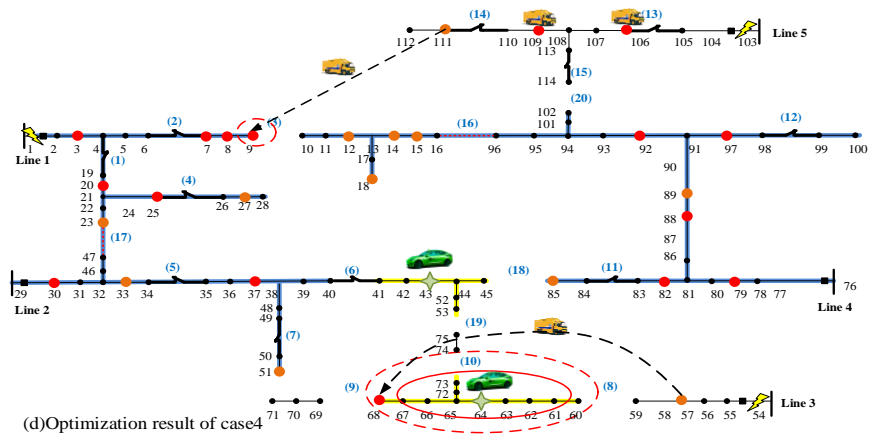

Fig. 5. Optimization results. (a) Case 1; (b) Case 2; (c) Case 3; (d) Case 4.

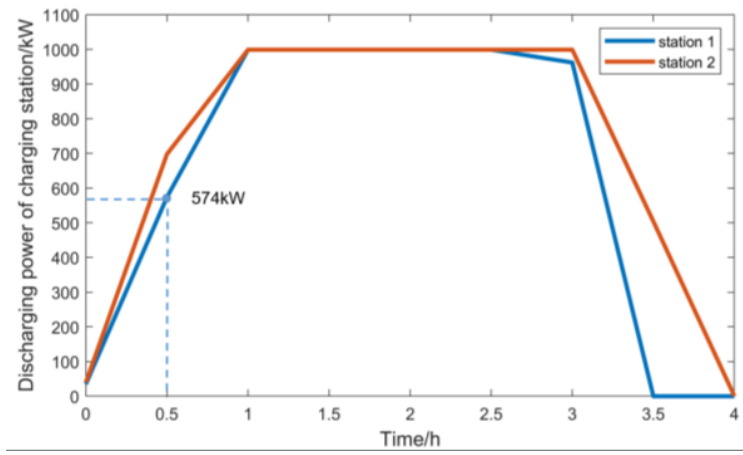

Fig. 6. Actual discharging power of the stations.

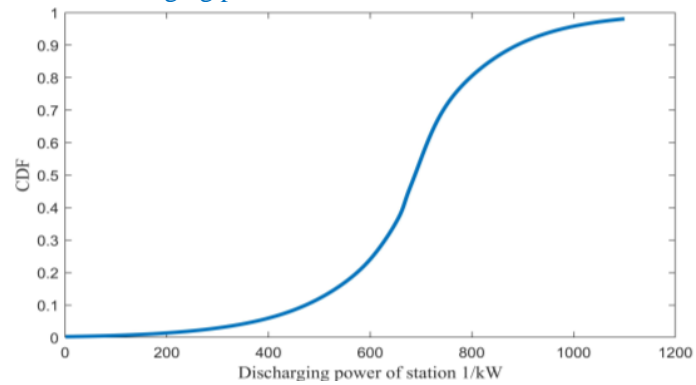

Fig. 7(a). CDF of the discharging power in Station 1

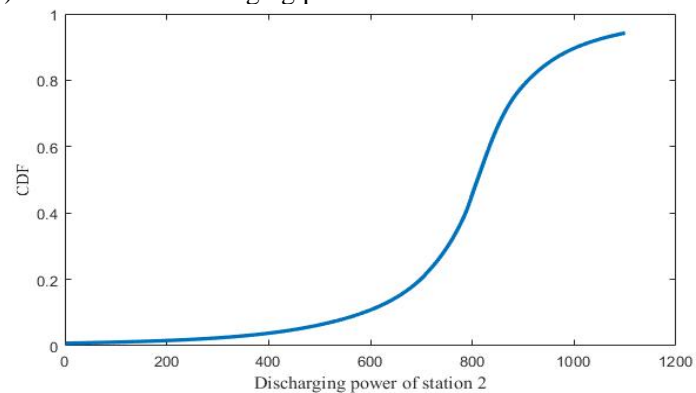

(b) Station 2

Fig. 7(b). CDF of the discharging power in Station 2. 
TABLE I

RESULTS OF TOPOLOGY AND EPVS OF DIFFERENT CASES

\begin{tabular}{|c|c|c|c|c|c|c|c|c|c|}
\hline \multirow{2}{*}{$\begin{array}{c}\text { Case } \\
\mathrm{s}\end{array}$} & \multirow{2}{*}{$\begin{array}{c}\text { Open } \\
\text { switches }\end{array}$} & \multicolumn{8}{|c|}{ Destination nodes of the EPVs } \\
\hline & & $10: 30$ & 11:00 & 11:30 & 12:00 & $12: 30$ & 13:00 & $13: 30$ & 14:00 \\
\hline 1 & $2,3,18,19,20$ & $8,68,108,106$ & $8,68,108,106$ & $8,68,108,106$ & $8,68,108,106$ & $8,68,108,106$ & $8,68,108,106$ & $8,68,108,106$ & $8,68,108,106$ \\
\hline 2 & $\begin{array}{c}3,8,9,10,18 \\
19,20 \\
\end{array}$ & $\begin{array}{c}111,109, \\
106,57 \\
\end{array}$ & $\begin{array}{c}111,109, \\
106,57 \\
\end{array}$ & $\begin{array}{c}111,109, \\
106,57 \\
\end{array}$ & $\begin{array}{c}111,109, \\
106,57 \\
\end{array}$ & $\begin{array}{c}111,109, \\
106,57 \\
\end{array}$ & $\begin{array}{c}111,109, \\
106,57 \\
\end{array}$ & $\begin{array}{c}111,109, \\
106,57 \\
\end{array}$ & $\begin{array}{c}111,109, \\
106,57 \\
\end{array}$ \\
\hline 3 & $\begin{array}{c}3,8,9,10,18 \\
19,20\end{array}$ & $\begin{array}{c}111,109, \\
106,57\end{array}$ & $\begin{array}{c}111,109, \\
106,57\end{array}$ & $\begin{array}{c}111,109, \\
106,57\end{array}$ & $\begin{array}{c}111,109, \\
106,57\end{array}$ & $\begin{array}{c}111,109, \\
106,57 \\
\end{array}$ & $\begin{array}{c}106,109,57, \\
\text { from } 111 \text { to } 9\end{array}$ & $106,109,9,57$ & $106,109,9,57$ \\
\hline 4 & $\begin{array}{c}3,8,9,10,18 \\
19,20\end{array}$ & $\begin{array}{c}106,109 \\
111,57\end{array}$ & $\begin{array}{c}106,109 \\
111,57\end{array}$ & $\begin{array}{c}106,109 \\
111,57\end{array}$ & $\begin{array}{c}106,109 \\
111,57\end{array}$ & $\begin{array}{c}106,109 \\
111,57\end{array}$ & $\begin{array}{c}106,109, \\
\text { from } 111 \text { to } \\
9, \\
\text { from } 57 \text { to } 68\end{array}$ & $106,109,9,68$ & $106,109,9,68$ \\
\hline
\end{tabular}

TABLE II

COMPARISON OF RECOVERED ELECTRICITY QUANTITY OF DIFFERENT CASES

\begin{tabular}{|c|c|c|c|c|c|c|c|c|c|c|}
\hline \multirow[t]{2}{*}{ Cases } & \multicolumn{2}{|c|}{$\begin{array}{l}\text { Electricity quantity restored by } \\
\text { main grid and station } 1(\mathrm{kWh})\end{array}$} & \multicolumn{2}{|c|}{$\begin{array}{c}\text { Electricity quantity } \\
\text { restored by EPVs (kWh) }\end{array}$} & \multicolumn{2}{|c|}{$\begin{array}{l}\text { Electricity quantity } \\
\text { restored by station } 2 \\
(\mathrm{kWh})\end{array}$} & \multicolumn{2}{|c|}{ Sum $(\mathbf{k W h})$} & \multicolumn{2}{|c|}{$\begin{array}{l}\text { Total recovery } \\
\quad \text { rate }(\%)\end{array}$} \\
\hline & Total load & Primary load & Total load & $\begin{array}{c}\text { Primary } \\
\text { load }\end{array}$ & Total load & $\begin{array}{c}\text { Primary } \\
\text { load }\end{array}$ & $\begin{array}{l}\begin{array}{l}\text { Total } \\
\text { load }\end{array} \\
\text { load }\end{array}$ & $\begin{array}{c}\text { Primary } \\
\text { load }\end{array}$ & $\begin{array}{l}\text { Total } \\
\text { load }\end{array}$ & $\begin{array}{c}\text { Primary } \\
\text { load }\end{array}$ \\
\hline Case 1 & 9735.71 & 1639.68 & 2894.07 & 2894.07 & I & I & 12629.78 & 4533.75 & 60.02 & 87.63 \\
\hline Case 2 & 10820.20 & 2723.97 & 2579.50 & 1459.54 & 1384.42 & 486.21 & 14783.91 & 4669.82 & 70.26 & 90.24 \\
\hline Case 3 & 10820.20 & 2723.97 & 2528.86 & 1598.79 & 1384.42 & 486.21 & 14733.33 & 4808.98 & 70.02 & 92.95 \\
\hline Case 4 & 10820.00 & 2723.97 & 2488.98 & 1795.16 & 1384.42 & 486.21 & 14693.40 & 5005.34 & 69.83 & 96.72 \\
\hline
\end{tabular}

\section{Superiority for Considering the Coordination of Network Reconfiguration and EPVs}

To verify the superiority of the coordination of network reconfiguration, EPVs and discharging stations, Case 5 and Case 6 are designed and compared with Case 4 proposed in this paper. The uncertainties of E-taxis are taken into account in all three cases.

Case 5 is the scheme considering network reconfiguration and E-taxis without EPVs.

Case 6 is the scheme considering network reconfiguration, EPVs and E-taxis, but reconfiguration and E-taxis are used for restoration first, and then EPVs are dispatched which will not move again in the whole restoration period.

The optimization result of case 5 is to break switches $3,8,9,13,14,16$ and 18 as shown in Fig. 8(a). Loads 60-68 and 72-73 in line 3 were restored by discharging station 2, which achieve an island recovery. Part of the loads in line 1 are restored by line 2 and part of loads in line 5 are restored by line 4 , because the restoration capability of Case 5 is limited, and the lines tend to restore the branches with more important load (i.e., branches of line 5).

In Case 6, EPVs have been dispatched to Nodes 12,15,57 and 111, shown in Fig. 8 (b), and 2548.49 kWh loads are further restored compared with Case 5. However, a continuous power supply for the primary loads cannot be guaranteed in this case. For example, the continuous power supply time for load 68 is only $2.5 \mathrm{~h}$ shown in Fig. 9. The reason is that the primary load of Node 68 has been shed when the load recovery range of the discharging Station 2 is reduced due to the reduction of battery capacities of E-taxis and EPVs are no longer moving during this period.

The total load and primary load power recovery of Case 5 is $644.25 \mathrm{kWh}$ and $335.52 \mathrm{kWh}$ respectively more than Case 6. That is because the utility grid can restore as many other loads as possible within the capabilities under the premise that EPVs can recover some important loads considering the coordination of network reconfiguration and EPVs. Moreover, continuous power supply time for the primary load 68 is increased to $3.5 \mathrm{~h}$, as shown in Fig. 9, because EPVs can quickly move and provide power supply for primary load when the restoration capability of discharging stations is insufficient and primary loads have to be shed.

Fig. 10 shows the primary load recovery rate structure in every recovery period. In Case 4, the green bars in Fig. 10(a) increase in periods 8 and 9. It is because that EPVs move during the recovery period and support the primary load 9 and load 68 which are shed by the main grid due to the reduced capacity of stations. In Case 6, EPVs are dispatched after the reconfiguration and lack coordination to move. Therefore, the primary load recovery capacity is worse than that in Case 4.

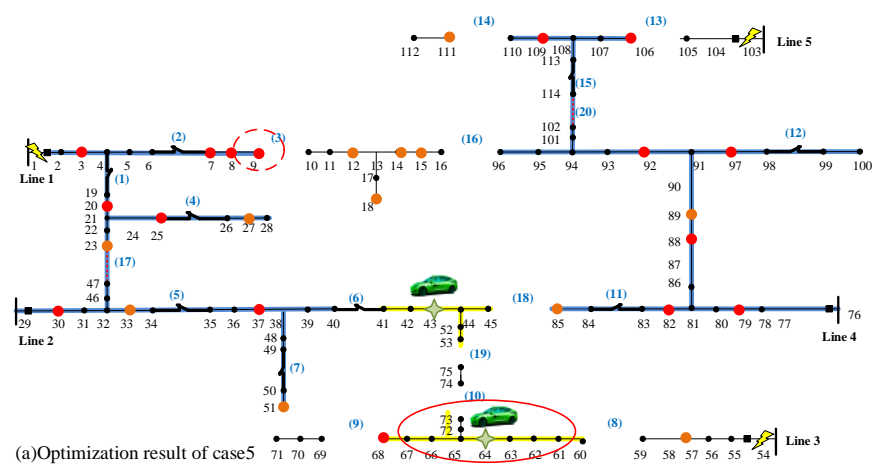




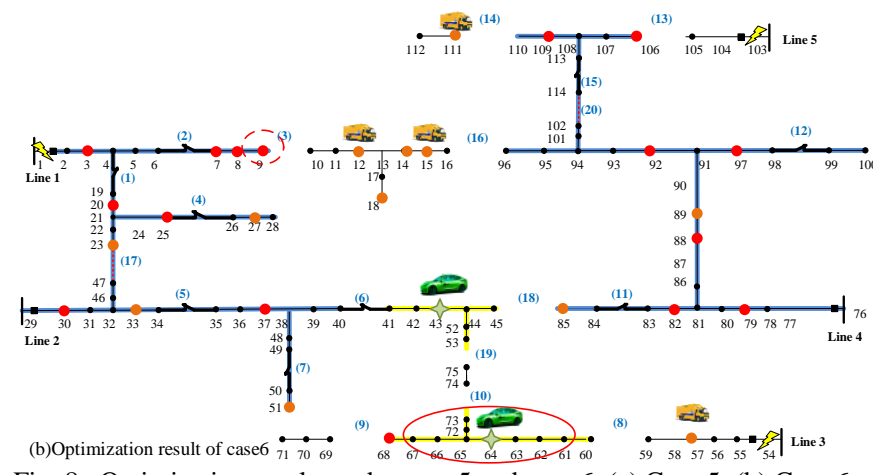

Fig. 8. Optimization results under case 5 and case 6. (a) Case 5; (b) Case 6.

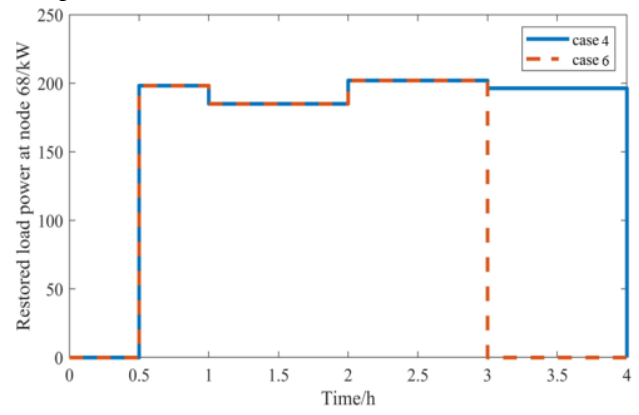

Fig. 9. The continuous power supply time for load 68.

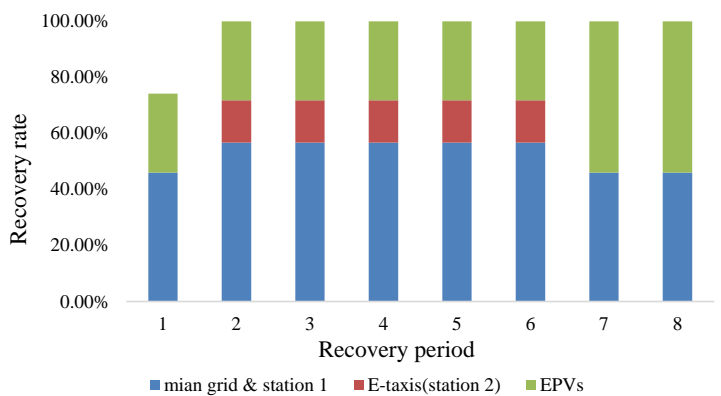

(a)

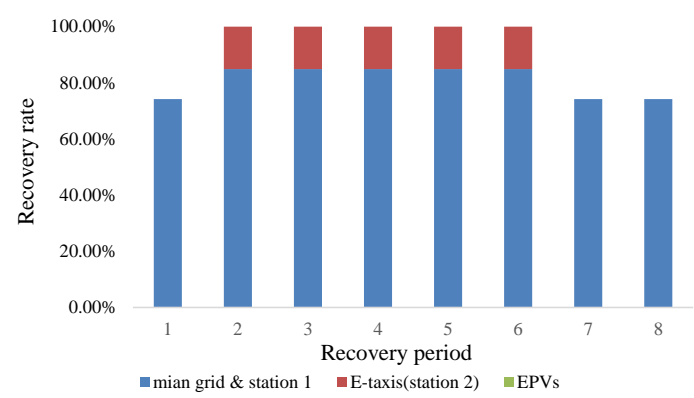

(b)

Fig. 10. Primary load recovery rate structure. (a) Case 4, (b) Case 6.

\section{A larger-scale system analysis using the proposed method}

A 9-feeder distributed network, which is modified based on a real network, is shown in Fig. 10. The maximum load power is $68007 \mathrm{~kW}+20402 \mathrm{kVar}$. The fault occurs at 10:00 am and lasts 4 hours and the dispatching time-scale is set as 0.5 hour. 400 E-taxis and $8 \mathrm{EPVs}$ are involved in the response process with the same condition as the case studies. The confidence level is set as 0.8 . The optimization results using the proposed method are shown in Table III.

TABLE III
RESULTS OF TOPOLOGY AND EPVS

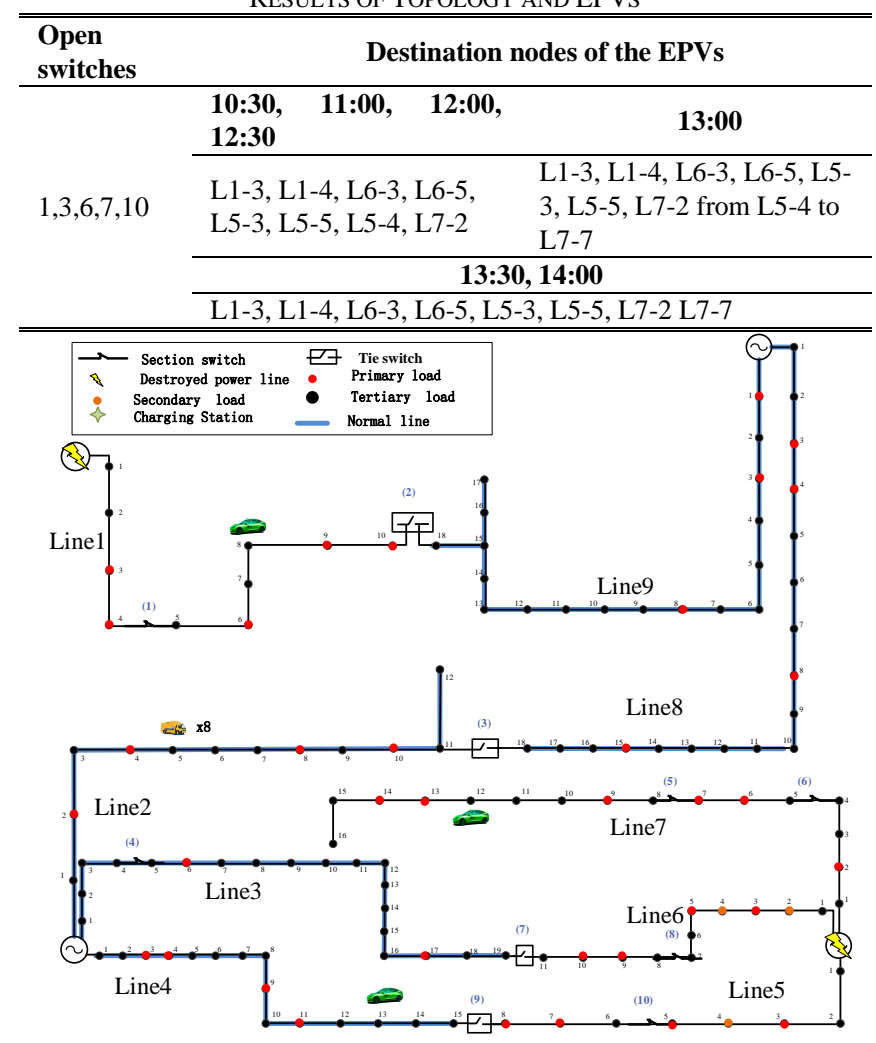

Fig 10. 9-feeder urban distribution system.

The total load and primary load power recovery is 65876.28 $\mathrm{kWh}$ and $38052.21 \mathrm{kWh}$, Electricity quantity restored by EPVs is $16245.69 \mathrm{kWh}$ including $14989.26 \mathrm{kWh}$ of primary load. Switch 5 opens after 12:30 because of the uncertainty of E-taxis. $70.3 \%$ of the total load and $96.2 \%$ of the primary load has been restored. The results show the scalability of the proposed method.

\section{CONCLUSIONS}

A coordinated restoration optimization method of EPVs and distribution network reconfiguration considering the uncertain restoration capabilities of discharging stations with E-taxis is proposed in this paper.

A joint probability distribution function has been established based on the Gaussian Mixture Model to describe the uncertainty of station discharging capabilities with E-taxis. The proposed method, which considers the uncertain restoration capabilities of discharging stations in the coordinated optimization of network reconfiguration and EPV dispatching, can lead to a more accurate restoration. The actual recovery capacity of primary loads with the proposed method is $7.2 \%$ higher than that in the case without considering the uncertainties of taxis.

The proposed coordinated restoration method can make full use of the restoration capabilities of EPVs and utility grids, because the utility grid can restore as many other loads as possible within the capabilities under the premise that EPVs can recover some important loads. Moreover, continuous power supply time for primary loads can be increased considering the coordination of network reconfiguration, EPVs and E-taxis, 
because EPVs can quickly move and provide power supply for primary load when the restoration capability of discharging stations is insufficient.

\section{REFERENCES}

[1] N. Safaei, D. Banjevic and A. K. S. Jardine, "Workforce Planning for Power Restoration: An Integrated Simulation-Optimization Approach," IEEE Trans. Power Syst., vol. 27, pp. 442-449, 2012.

[2] L. Zhang, B. Tong, Z. Wang, W. Tang and C. Shen, "Optimal Configuration of Hybrid AC/DC Distribution Network Considering the Temporal Power Flow Complementarity on Lines," IEEE Trans. Smart Grid, early access, doi: 10.1109/TSG.2021.3102615.

[3] Z. Wang and J. Wang, "Self-Healing Resilient Distribution Systems Based on Sectionalization Into Microgrids," IEEE Trans. Power Syst., vol 30, pp. 3139-3149, 2015.

[4] Y. Xu, Y. Wang, J. He, M. Su, and P. Ni, "Resilience-Oriented Distribution System Restoration Considering Mobile Emergency Resource Dispatch in Transportation System," IEEE Access, vol. 7, pp. 73899-73912, 2019.

[5] S. Yao, P. Wang and T. Zhao, "Transportable Energy Storage for More Resilient Distribution Systems With Multiple Microgrids," IEEE Trans. Smart Grid, vol. 10, pp. 3331-3341, 2019.

[6] Y. Lu, Y. Liang, Z. Ding*, Q. Wu, T. Ding, W. Lee. Deep Reinforcement Learning based Charging Pricing for Autonomous Mobility-on-Demand, Transactions on Smart Grid, 2021, Accepted. DOI: 10.1109/TSG.2021.3131804.

[7] P. Jamborsalamati, M. J. Hossain, S. Taghizadeh, G. Konstantinou, M. Manbachi and P. Dehghanian, "Enhancing Power Grid Resilience Through an IEC61850-Based EV-Assisted Load Restoration," IEEE Trans. Ind. Informat., vol. 16, no. 3, pp. 1799-1810, March 2020.

[8] Z. Ding, J. Guo, K. Lai and W. -J. Lee, "Spatial-Temporal Demand Management and Benefit Allocation for Geo-Distributed Charging Station and EV Aggregators," IEEE Trans. Ind Appl., vol. 56, no. 6, pp. 6238-6249, Nov.-Dec. 2020.

[9] F. Wang et al., "A Multi-Stage Restoration Method for Medium-Voltage Distribution System With DGs," IEEE Trans. Smart Grid, vol. 8, no. 6, pp. 2627-2636, Nov. 2017.

[10] W. Chen et al., "Robust Islanded Restoration Coordinating Multiple Distributed Resources to Enhance Resilience of Active Distribution System," 2019 IEEE Power \& Energy Society General Meeting (PESGM), Atlanta, GA, USA, 2019, pp. 1-5.

[11] P. Li, M. Yang, et al, "Robust Decentralized Coordination of Transmission and Active Distribution Networks," IEEE Trans. Industry Applications, in press.

[12] B. Zeng, H. Dong, et al, "Bilevel Robust Optimization of Electric Vehicle Charging Stations With Distributed Energy Resources," IEEE Trans. Industry Applications, vol. 56, pp. 5836-5847, 2020.

[13] P. Li, M. Yang, et al, "Confidence Interval Based Distributionally Robust Real-Time Economic Dispatch Approach Considering Wind Power Accommodation Risk," IEEE Trans. Sustainable Energy, vol. 12, pp. 5869, 2021

[14] P. Li, Q. Wu, et al, "Distributed Distributionally Robust Dispatch for Integrated Transmission-Distribution Systems," IEEE Trans. Power Systems, vol. 36, pp. 1193-1205, 2021.

[15] S. Sharma, K. R. Niazi, K. Verma and T. Rawat, "Impact of battery energy storage, controllable load and network reconfiguration on contemporary distribution network under uncertain environment," IET Gener. Transm. Distrib., vol. 14, no. 21, pp. 4719-4727, Sept. 2020.

[16] L. Sun, X. Wang, W. Liu, Z. Lin, F. Wen, S. P. Ang, and M. A. Salam, "Optimisation model for power system restoration with support from electric vehicles employing battery swapping," IET Gener. Transm. Distrib., vol. 10, no. 3, pp. 771-779, July 2016.

[17] D. Tang and P. Wang, "Probabilistic Modeling of Nodal Charging Demand Based on Spatial-Temporal Dynamics of Moving Electric Vehicles," IEEE Trans. Smart Grid, vol. 7, no. 2, pp. 627-636, 2016.

[18] D. Tang and P. Wang, "Nodal Impact Assessment and Alleviation of Moving Electric Vehicle Loads: From Traffic Flow to Power Flow," IEEE Trans. Power Syst., vol. 31, pp. 4231-4242, 2016.

[19] S. Yao, P. Wang, X. Liu, H. Zhang, and T. Zhao, "Rolling Optimization of Mobile Energy Storage Fleets for Resilient Service Restoration," IEEE Trans. Smart Grid, vol. 11, pp. 1030-1043, 2020.

[20] H. I. Kang, B. Lee and K. Kim, "Path Planning Algorithm Using the Particle Swarm Optimization and the Improved Dijkstra Algorithm,"
2008 IEEE Pacific-Asia Workshop on Computational Intelligence and Industrial Application, Wuhan, China, 2008, pp. 1002-1004.

[21] L. Vanhaverbeke, D. Schreurs, Q. De Clerck, M. Messagie and J. Van Mierlo, "Total cost of ownership of electric vehicles incorporating Vehicle to Grid technology," 2017 Twelfth International Conference on Ecological Vehicles and Renewable Energies (EVER), 2017, pp. 1-6, doi: 10.1109/EVER.2017.7935931.

[22] Z Wang, C Shen, Y Xu, F Liu, X Wu and C Liu, "Risk-Limiting Load Restoration for Resilience Enhancement With Intermittent Energy Resources," IEEE Trans. Smart Grid, vol. 10, no. 3, pp. 2507-2522, 2019.

[23] X. Shen, M. Shahidehpour, Y. Han, S. Zhu, and J. Zheng, "Expansion Planning of Active Distribution Networks With Centralized and Distributed Energy Storage Systems," IEEE Trans. Sustain. Energy, vol. 8, pp. 126-134, 2017.

[24] R. A. Jabr, R. Singh and B. C. Pal, "Minimum Loss Network Reconfiguration Using Mixed-Integer Convex Programming," IEEE Trans. Power Syst., vol. 27, pp. 1106-1115, 2012.

[25] H. Duan, Q. Luo, Y. Shi, and G. Ma, "Hybrid Particle Swarm Optimization and Genetic Algorithm for Multi-UAV Formation Reconfiguration," IEEE Computational Intelligence Magazine, vol. 8, pp. 16-27, 2013.

[26] G. Carpinelli, G. Celli, S. Mocci, F. Mottola, F. Pilo, and D. Proto, "Optimal Integration of Distributed Energy Storage Devices in Smart Grids," IEEE Trans. Smart Grid, vol. 4, pp. 985-995, 2013.

[27] C. Lin, D. Deng, C. Kuo and Y. Liang, "Optimal Charging Control of Energy Storage and Electric Vehicle of an Individual in the Internet of Energy With Energy Trading," IEEE Trans. Ind. Infomat., vol. 14, no. 6, pp. 2570-2578, June 2018.

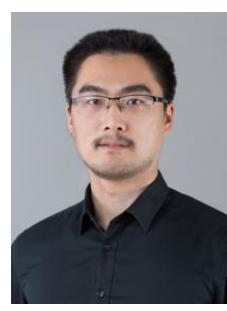

Lu Zhang (S'13-M'17) received his B.S. degree in electrical engineering and his Ph.D. degree in Agricultural Electrification and Automation from China Agricultural University, Beijing, China, in 2011 and 2016, respectively. He was a postdoc in the Department of Electrical Engineering at Tsinghua University from 2017 to 2019. He is currently an Associate Professor at the College of Information and Electrical Engineering, China Agricultural University, Beijing, China. His main research interests include hybrid AC/DC distribution network, renewable energy generation, and resilience.

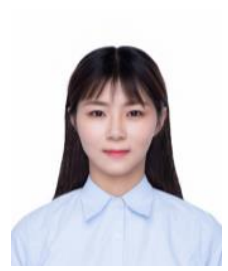

Shujun Jiang received the B.Eng. degree in Electrical Engineering and Automation from China Agricultural University, Beijing, China, in 2020 and is studying for the M.Sc. degree in Advanced Electrical Power System Engineering from the University of Manchester, U.K., from 2020. Her research interests include smart grid, renewable energy, electric vehicles and distribution network restoration.

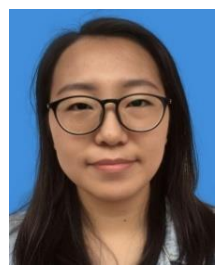

Bo Zhang received the B.S. degree in Electrical Engineering from China Agricultural University, Beijing, China, in 2016. She is currently working toward the Ph.D. degree at the College of Information and Electrical Engineering, China Agricultural University. Her main research interests include hybrid $\mathrm{AC} / \mathrm{DC}$ distribution network, renewable energy generation, and economic operation of active distribution network.

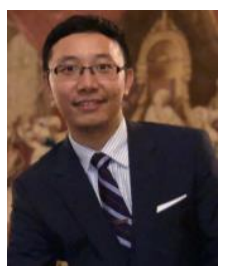

Gen Li (M'18) received the B.Eng. degree in Electrical Engineering from Northeast Electric Power University, Jilin, China, in 2011, the M.Sc. degree in Power Engineering from Nanyang Technological University, Singapore, in 2013 and the Ph.D. degree in Electrical Engineering from Cardiff University, Cardiff, U.K., in 2018.

From 2013 to 2016, he was a Marie Curie Early Stage Research Fellow funded by the European Commission's MEDOW project. He has been a Visiting Researcher at China Electric Power Research Institute and Global Energy Interconnection Research Institute, Beijing, China, at Elia, Brussels, Belgium and at Toshiba International (Europe), 
London, U.K. He has been a Research Associate at the School of Engineering, Cardiff University since 2017. His research interests include control and protection of HVDC and MVDC technologies, power electronics, reliability modelling and evaluation of power electronics systems.

Dr. Li is a Chartered Engineer in the U.K. He is an Associate Editor of the CSEE Journal of Power and Energy Systems. He is an Editorial Board Member of CIGRE ELECTRA. He is an IET Professional Registration Advisor. His Ph.D. thesis received the First CIGRE Thesis Award in 2018. He is the ViceChair of IEEE PES Young Professionals and the Technical Panel Sectary of CIGRE UK B5 Protection and Automation.

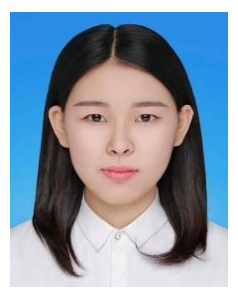

Zhaoqi Wang received the B.S. degree and the M.S. degree in electrical engineering from China Agricultural University, Beijing, China, in 2018 and 2020. She is currently working toward the Ph.D. degree at the College of Information and Electrical Engineering, China Agricultural University.

Her main research interests include power system resilience and modeling methods of uncertainty factors.

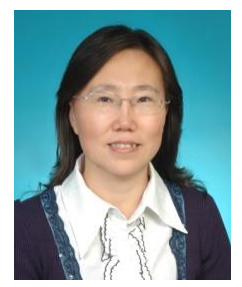

Wei Tang (M'13), the corresponding author of this paper, received the B.Sc. degree from Huazhong University of Science and Technology, Wuhan, China, in 1992 and the Ph.D. degrees from Harbin Institute of Technology, Harbin, China, in 1998.

From 1998 to 2000, she was a post-doctor with Harbin Engineering University. Currently she is a professor at College of Information and Electrical Engineering, China Agricultural University, Beijing, China. Her research interests include Distribution network economic and security operation, Distributed generation and Active distribution network. 